\title{
Fluoxetine for Motor Recovery After Acute Ischemic Stroke: A Randomized Placebo-Controlled Trial
}

\author{
Mahfuzur Rahman Babul ${ }^{1}$, Hassanuzzaman ${ }^{2}$, Zaman Ahammed $^{2}$, Anwarul Kibria ${ }^{3}$, Golam Faruk ${ }^{3}$, \\ Akramul Azam ${ }^{4}$, Mizanur Rahman ${ }^{5, *}$ \\ ${ }^{1}$ Medical Assistant Training School, Noakhali, Bangladesh \\ ${ }^{2}$ Department of Neurology, Chittagong Medical College Hospital, Chittagong, Bangladesh \\ ${ }^{3}$ Department of Anatomy, Chittagong Medical College, Chittagong, Bangladesh \\ ${ }^{4}$ Department of Pharmacology, Rangamati Medical College, Chittagong, Bangladesh \\ ${ }^{5}$ Department of Biochemistry, Rangamati Medical College, Chittagong, Bangladesh \\ Email address: \\ mizan2011bio@gmail.com (M. Rahman) \\ *Corresponding author
}

\section{To cite this article:}

Mahfuzur Rahman Babul, Hassanuzzaman, Zaman Ahammed, Anwarul Kibria, Golam Faruk, Akramul Azam, Mizanur Rahman. Fluoxetine for Motor Recovery After Acute Ischemic Stroke: A Randomized Placebo-Controlled Trial. American Journal of Psychiatry and

Neuroscience. Vol. 5, No. 3, 2017, pp. 31-36. doi: 10.11648/j.ajpn.20170503.11

Received: April 3, 2017; Accepted: April 19, 2017; Published: May 31, 2017

\begin{abstract}
Hemiplegia and hemiparesis are the most common deficits caused by stroke. A few small clinical trials suggest that Fluoxetine enhances motor recovery but its clinical efficacy is unknown in our setting. A randomized placebo-controlled trial was conducted at Neurology and medicine ward of Chittagong Medical College Hospital from 1/1/2013 to 31/12/2013. One hundred and twenty eight patients, aged between 40-60 years with a diagnosis of acute ischaemic stroke who fulfilled the defined inclusion and exclusion criteria were selected for the study. Eligible patients were randomly assigned, using a simple lottery method, in a 1:1 ratio to Fluoxetine (20 mg once per day, orally) or placebo group for 3 months starting within 10 days after the onset of stroke. The primary outcome was the mean change in the Rivermead Mobility Index (RMI) score between inclusion (day 0) and day 90. RMI score was significantly changed from D 0 to D 90 in Fluoxetine group in comparison to placebo group $(\mathrm{p}<.001)$ and mean $( \pm \mathrm{SD})$ improvement of RMI score in 90 days were significantly greater in the Fluoxetine group, than in the placebo group $[7.08( \pm 3.26)$ vs. $4.40( \pm 2.53)]$. Patients treated with Fluoxetine were nearly 3.2 times more likely to show motor improvement than placebo group and the NNT was 2. In patients with acute ischaemic stroke and with motor deficit, the early administration of Fluoxetine with physiotherapy enhanced motor recovery after 3 months.
\end{abstract}

Keywords: Fluoxetine, Ischaemic Stroke, Rivermead Mobility Index (RMI), NNT: Number Needed to Treat

\section{Introduction}

Stroke is caused by either a sudden reduction in the blood supply to the brain or by a hemorrhage. An acute stroke refers to the first 24-hour-period of a stroke event. Most strokes $(87 \%)$ are ischaemic and the rest (13\%) are hemorrhagic. Stroke is the second leading cause of disability in Europe after ischaemic heart disease (IHD) and is the sixth leading cause worldwide. The prevalence of stroke events is expected to increase across the globe as the global population aged over 65 increases $[1,2]$. Motor deficits are common after stroke ( $82 \%$ of patients) and are linked with reduced quality of life [3]. At 6 months after stroke, $65 \%$ of patients are unable to incorporate a paretic hand effectively into daily activities, which affects subjective well-being. Moreover, even when neurological examination declares the patient wholly recovered, $71 \%$ of patients report persistent motor deficits when studied using patient-reported outcomes. Lower extremity motor status is also linked with disability level. Only $37 \%$ of persons with stroke can walk after the first week post stroke, after which gait improvement is linked to better quality of life. Hemiplegic patients rank recovery of gait as their top priority [4].

Among the therapeutic strategies under study to improve 
long-term outcome after stroke are drugs targeting events that underlie recovery. Drugs that enhance recovery are separate from those that promote neuroprotection or reperfusion in patients with stroke. The value of specific rehabilitation therapies aimed at assisting adaptation to impairment is now well recognized, but therapeutic strategies designed to restore function by minimizing impairment are by comparison poorly developed. Recovery-based drugs have distinct therapeutic targets that are related to plasticity and growth after stroke, and in general, improvements in behavioral outcome are not accompanied by a reduction in infarct volume. Till now, there are no drugs approved in the United States to enhance motor recovery after stroke. For at least 2 classes of drug, serotonergic and dopaminergic, both of which are monoaminergic, existing evidence from human studies supports the possibility for enhancing motor outcome after stroke.

Serotonin normally plays a role in modulating multiple cognitive functions, particularly response inhibition and memory consolidation, and modulates the impact of punishment-related signals on learning and emotion [5-7]. Recent reports suggest potential clinical utility of selective serotonin reuptake inhibitor (SSRI) drugs for promoting improved motor outcome after stroke. Building on several prior smaller studies, [8-10] the Fluoxetine for Motor Recovery after Acute Ischaemic Stroke (FLAME) study was a double-blind, placebo-controlled trial that enrolled non depressed hemiplegic/hemiparetic patients within 10 days of ischaemic stroke onset. Patients were randomized to 3 months of oral fluoxetine $(20 \mathrm{mg} / \mathrm{d})$ or placebo. Patients randomized to fluoxetine showed significantly greater gains on the primary end point, change in the arm/leg Fugl-Meyer motor score to day $90(P=0.003)$, a remarkable difference of 9.7 points on this 100 -point scale [11].

Several different mechanisms might account for these findings. The central mechanism of action for SSRI drugs in the treatment of major depression is via their high affinity for the serotonin transporter; drug binding to the transporter inhibits serotonin removal from the synaptic cleft, with longterm SSRI administration down regulating and desensitizing key serotonin receptors, thereby dampening negative feedback on serotonin release [12]. Other suggested mechanisms of action for SSRI drugs include reducing neural inflammation, enhancing neurotrophin activity, and increasing eurogenesis. Chronic SSRI dosing increases intracortical facilitation and reduces intracortical inhibition, and these changes have been compared with reinstating conditions of developmental critical periods. In addition, serotonin modulates spinal motor control through multiple effects on spinal motor circuits, including regulation of rhythmic activity and control of excitability, by acting on intrasynaptic and extrasynaptic receptors; this may help locomotor function [13-16]. Motor excitability is increased in both hemispheres in stroke patients during motor recovery, In particular, it has been suggested that antidepressant drugs can modulate motor excitability. Acler $\mathrm{M}$ et al. investigated motor area excitability in patients with stroke after oral administration of citalopram through transcranial magnetic stimulation. Their findings suggest that treatment with serotonergic drugs can bring about a significant decrease of the motor cortex excitability in stroke patients with effects on both the affected and unaffected hemispheres associated with a better motor recovery [17].

There is very limited number of study regarding evaluation of long term use of Fluoxetine over motor function among stroke patients in the context of Bangladesh. So it is a good opportunity to do a study in this context. The aim of this study was to test whether a 3-month treatment with Fluoxetine would enhance motor recovery when given early after an ischaemic stroke to patients with moderate to severe motor deficits.

Pharmacology of Fluoxetine [18, 19].

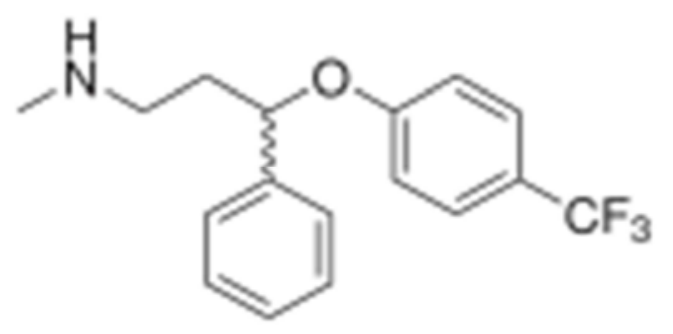

Fluoxetine is the prototypic drug of the group of selective serotonin reuptake inhibitors (SSRIs) which are chemically diverse antidepressant that specifically inhibit serotonin reuptake, having 300- to 3000-fold greater selectivity for the serotonin transporter, as compared to the norepinephrine transporter.

The SSRIs block the reuptake of serotonin, leading to increased concentrations of the neurotransmitter in the synaptic cleft and, ultimately to greater postsynaptic neuronal activity. These drugs do not usually produce central nervous system (CNS) stimulation or mood elevation in normal individuals.

The primary indication for Fluoxetine is depression. A number of other psychiatric disorders also respond favorably to Fluoxetine, including obsessive-compulsive disorder, panic disorder, generalized anxiety disorder, posttraumatic stress disorder, social anxiety disorder, premenstrual disorder, and bulimia nervosa.

Adverse effects: Although the Fluoxetine is considered to have fewer and less severe adverse effects than the TCAs and MAOIs, the Fluoxetine is not without troublesome adverse effects, such as headache, sweating, anxiety and agitation, gastrointestinal (GI) effects (nausea, vomiting, diarrhea), weakness and fatigue, sexual dysfunction, changes in weight, sleep disturbances (insomnia and somnolence), and the above-mentioned potential for drug-drug interactions.

Action on stroke patient: Fluoxetines have been used in the treatment of stroke patients, including those with and without symptoms of depression. In a study, Lim CM et al. tested whether Fluoxetine protects neuronal death in a rat cerebral ischemia model of middle cerebral artery occlusion (MCAO). The administration of Fluoxetine intravenously $(10 \mathrm{mg} / \mathrm{kg})$ at $30 \mathrm{~min}, 3 \mathrm{hr}$, or $6 \mathrm{hr}$ after MCAO reduced infarct volumes to 
$21.2 \pm 6.7 \%, 14.5 \pm 3.0 \%$, and $22.8 \pm 2.9 \%$, respectively, of that of the untreated control. Moreover, the neuroprotective effect of Fluoxetine was evident when it was administered as late as $9 \mathrm{hr}$ after $\mathrm{MCAO} /$ reperfusion. These neuroprotective effects were accompanied by improvement of motor impairment and neurological deficits. The Fluoxetine-treated brain was found to show marked repressions of microglia activation, neutrophil infiltration, and proinflammatory marker expressions. Moreover, Fluoxetine suppressed NF- $\kappa B$ activity dose-dependently in the post ischaemic brain and also in lipopolysaccharide-treated primary microglia and neutrophil cultures, suggesting that $\mathrm{NF}-\kappa \mathrm{B}$ activity inhibition explains in part its anti-inflammatory effect. These results demonstrate that curative treatment of Fluoxetine affords strong protection against delayed cerebral ischaemic injury, and that these neuroprotective effects might be associated with its anti-inflammatory effects [20].

\section{Material and Methods}

A randomized placebo controlled trial was conducted from $1 / 1 / 2013$ to $31 / 12 / 2013$. Patients who had an acute ischaemic stroke within 10 days after the incidence that cause hemiparesis or hemiplegia with radiologically verified lesion get admitted to neurology and medicine ward or referred to Neurology OPD of CMCH.

Selection criteria included radiologically (C. T scan of Head) documented ischaemic stroke, have definite motor deficit- hemiplegia, age between 40 to 60 years and ischaemic stroke within 10 days. Exclusion criteria are $\mathrm{H} / \mathrm{O}$ previous stroke, patient with known depressive illness and on antidepressant, global or sensory Aphasia(Masking detection and assessment of depression), unconscious patients, terminally ill patient, having other severe medical(IHD), or pregnancy which may prevent follow-up. Study populations were selected after fulfilling the selection criteria. All patients were informed about the treatment options and written witnessed consent was taken from the patients or attendance whichever was applicable. Then the patients were allocated randomly to group A and group B. Group A patient was given placebo with physiotherapy and group B patients was given Fluoxetine with physiotherapy. Patient who died or withdrawn from the study within the study period were considered as dropped out. Then assessment of motor functional deficit at base line using Rivermead Mobility Index scale was done and improvement was assessed on follow up. During assessment of motor recovery patient was assessed in relation with movement in bed, lying to seating capacity, standing ability, walking ability, picking ability, bathing and running capacity. Follow up was done on day 30 and day 90. Mobile phone or telephone number of the patient or first degree relatives was preserved for further communication.

\section{Data Processing and Analysis}

All the data were checked and edited after collection. The primary outcome was analyzed using the intention to treat (ITT) method. The full analysis set for the ITT method was include all randomized subjects, regardless of their subsequent withdrawal after enrollment. Continuous variables were reported as the means $\pm \mathrm{SD}$, and categorical variables were reported as percentages. Baseline characteristics were compared by either Student's $t$-test for continuous variables or the $\chi^{2}$ test (Fisher's exact test when the expected value is $<5$ ) for categorical data. The primary analysis for efficacy (full-analysis set) was consisted of a comparison of the change in RMI scores at 90 days. To handle the missing data derived from withdrawal and lost to follow-up patients, the series mean value was used for the analysis. A binary logistic regression analysis was performed to control for baseline factors. Statistical significance was defined as $\mathrm{P}<0.05$ and confidence interval set at 95\% level. SPSS (Statistical Package for Social Science) for Windows version 15.0 software was used for the analyses.

\section{Result and Observation}

\subsection{Patterns of Risk Factors Among the Patients in Respect to Study Group}

In respect to risk factors of ischaemic stroke both the study groups are similar. There was no significant difference between Fluoxetine and placebo groups in respect of presence or absence of HTN, DM, smoking history, hyperlipidaemia, family history of stroke (Table 1).

Table 1. Pattern of risk factors among the study groups.

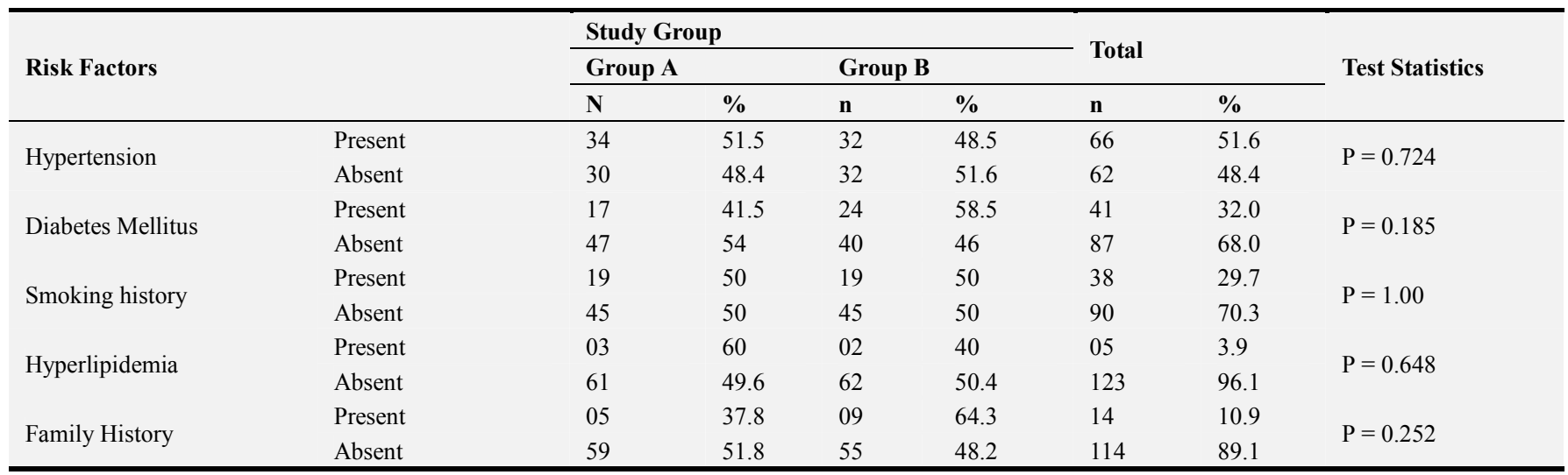




\subsection{Patterns of Neurological Findings Among the Patients in Respect to Study Group}

In respect to neurological findings at presentation both the study groups are similar. There was no significant difference between Fluoxetine and placebo groups in respect to Speech, higher cerebral function and motor function (Table 2).

Table 2. Distribution of neurological findings among the study groups.

\begin{tabular}{|c|c|c|c|c|c|c|c|c|}
\hline \multirow{3}{*}{ Neurological Findings } & & \multicolumn{4}{|c|}{ Study Group } & \multirow{2}{*}{\multicolumn{2}{|c|}{ Total }} & \multirow{3}{*}{ Test statistics } \\
\hline & & \multicolumn{2}{|c|}{ Group A } & \multicolumn{2}{|c|}{ Group B } & & & \\
\hline & & $\mathbf{N}$ & $\%$ & $\mathbf{N}$ & $\%$ & $\mathbf{n}$ & $\%$ & \\
\hline \multirow{3}{*}{ Speech } & Normal & 49 & 52.7 & 44 & 47.3 & 93 & 72.7 & \multirow{3}{*}{$\mathrm{P}=0.586$} \\
\hline & Aphasia & 06 & 40.0 & 09 & 60.0 & 15 & 11.7 & \\
\hline & Dysarthria & 09 & 45.0 & 11 & 55.0 & 20 & 15.6 & \\
\hline \multirow{2}{*}{ Higher Cerebral Function } & Oriented & 64 & 50.0 & 64 & 50.0 & 128 & 100.0 & \multirow{2}{*}{$* *$} \\
\hline & Disoriented & 00 & 00 & 00 & 00 & 00 & 0.0 & \\
\hline Motor Difficulties & Absent & 00 & 00 & 00 & 00 & 00 & 0.0 & $* *$ \\
\hline
\end{tabular}

** No statistics were calculated because Higher Cerebral Function \& Motor Difficulties are constant.

\subsection{Improvement of Motor Function in Relation to Study Groups}

Table 3 shows the difference of motor recovery in Fluoxetine and placebo groups at day 0, 30 and 90 days.
There is statistically significant difference of the mean RMI scores between Fluoxetine and placebo group at 90 days. The Effect Size by standardized Mean Difference (SMD) was 0.55 (Moderate Effect Size as per Cohen's d).

Table 3. Rivermead mobility index at Day 0, 30 \& 90 among the study groups.

\begin{tabular}{lllll}
\hline & & MEAN & 土 SD & PANGE \\
\cline { 2 - 5 } Rivermead Mobility Index & Group A $(\mathrm{n}=64)$ & 0.89 & 1.74 & $0-10$ \\
At Day 0 & Group B(n=64) & 1.23 & 1.67 & $0-7$ \\
& TOTAL & 1.06 & 1.71 & $0-10$ \\
& & MEAN & \pm SD & RANGE \\
Rivermead Mobility Index & Group A(n=54) & 2.43 & 2.64 & $0-15$ \\
At Day 30 & Group B(n=56) & 3.34 & 2.36 & $1-11$ \\
& TOTAL & 2.89 & 2.53 & $0-15$ \\
Rivermead Mobility Index & Group A(n=50) & MEAN & \pm SD & 2.058 \\
At Day 90 & Group B(n=49) & 4.40 & 2.53 & $2-15$ \\
& TOTAL & 7.08 & 3.26 & $3-14$ \\
\hline
\end{tabular}

\subsection{Pattern of Muscle Power Improvement Among the Study Group}

Muscle power is significantly improve in Fluoxetine group both at day 30 and day 90 in comparison to inclusion day, but not in placebo group (Table 4).

Table 4. Changes of muscle power over time in relation with day 0.

\begin{tabular}{llllll}
\hline Group & Evaluation day & Muscle power & N & Mean & PSD \\
\hline & Day 30 & Score $\leq 2$ & 43 & 0.49 & 0.45 \\
B & $(\mathrm{n}=56)$ & Score $>2$ & 13 & 2.38 & 0.165 \\
(Fluoxetine) & Day 90 & Score $\leq 2$ & 36 & 0.88 & 0.07 \\
& $(\mathrm{n}=49)$ & Score $>2$ & 13 & 2.00 & 1.335 \\
& Day 30 & Score $\leq 2$ & 47 & 1.70 & 0.267 \\
A & $(\mathrm{n}=54)$ & Score $>2$ & 7 & 2.00 & 0.225 \\
(Placebo) & Day 90 & Score $\leq 2$ & 40 & 1.82 & 0.353 \\
& $(\mathrm{n}=50)$ & Score $>2$ & 10 & 2.33 & .063 \\
\hline
\end{tabular}

\subsection{Calculation of Effect Size Measures (Interest in Favorable Cases)}

Table 5. Motor improvement between Fluoxetine vs. placebo group.

\begin{tabular}{|c|c|c|c|c|}
\hline \multirow{2}{*}{ Patient category } & \multicolumn{2}{|c|}{ Motor improvement at 90 days } & \multirow{2}{*}{ Lost to follow-up } & \multirow{2}{*}{ Total } \\
\hline & $>4$ in $\mathrm{RMI}$ & $\leq 4$ in $\mathrm{RMI}$ & & \\
\hline Fluoxetine & 39 (a) & $10(\mathrm{~b})$ & 15 & 64 \\
\hline Placebo & $12(\mathrm{c})$ & $34(d)$ & 14 & 64 \\
\hline
\end{tabular}




\begin{tabular}{|c|c|c|c|c|c|c|}
\hline Case analyzed & $E R R * a /(a+b)$ & $C E R * c /(c+d)$ & ABI/ARR* (ERR-CER) & NNT* (1/ARR) & RR* (ERR/CER) & RRR* (1-RR) \\
\hline \multicolumn{7}{|c|}{ A. Base case: Ignoring loss to follow-up cases } \\
\hline & $79.59 \%$ & $24 \%$ & $55.59 \%$ & $1.8(2)$ & 3.32 & -2.32 \\
\hline \multicolumn{7}{|c|}{ B. Worst case: Lost experimental subjects do poorly and control subjects do well } \\
\hline & $60.94 \%$ & $40.63 \%$ & $20.31 \%$ & $4.9(5)$ & 1.49 & -.51 \\
\hline \multicolumn{7}{|c|}{ C. Intention to treat analysis: Replacing the missing value by series mean } \\
\hline & $84 \%$ & $38 \%$ & $46 \%$ & $2.2(3)$ & 2.21 & 1.22 \\
\hline
\end{tabular}

ERR: Experimental Event Rate, CER: Control Event Rate, ARR/ ABI: Absolute Risk Reduction/ Absolute Benefit Increase, NNT: Number Needed to Treat, RR: Relative Risk, RRR: Relative Risk Reduction.

\section{Discussion}

A positive effect on motor recovery was noted in patients with acute ischaemic stroke who were treated with Fluoxetine for three months in the present study. This effect was assessed as a change in RMI score between day 0 and day 90. Recently, the use of antidepressant drugs as an adjunctive treatment to augment recovery in stroke patients has been studied in small clinical trials $[8,10,17,2]$. Fluoxetine is a selective serotonin reuptake inhibitor that is widely used in the treatment of major depression including after stroke. It was established in different preclinical studies that Fluoxetine protects neuronal death following ischemia. These neuroprotective effects were accompanied by improvement of motor impairment and neurological deficits. The Fluoxetine-treated brain was found to show marked repressions of microglia activation, neutrophil infiltration, and proinflammatory marker expressions. Moreover, fluoxetine suppressed NF-kappa B activity dose-dependently in the post ischaemic brain and also in lipopolysaccharidetreated primary microglia and neutrophil cultures, suggesting that NF-kappa B activity inhibition explains in part its antiinflammatory effect. These results demonstrate that curative treatment of Fluoxetine affords strong protection against delayed cerebral ischemic injury, and that these neuroprotective effects might be associated with its antiinflammatory effects [22].

In 2011, Chollet and colleagues tested adjunctive treatment with Fluoxetine in addition to physical rehabilitation to improve motor recovery from cerebral infarct, with excellent results [11]. In the present study, intervention and control groups were similar in respect of sex, age, locality, nature of work and socioeconomic status. In a study done in Chittagong Bangladesh by Kyastagir et al. found among 59 study patients, male patient was $39(66.6 \%)$ and female was 20 (33.3\%). [23] In Bangladesh usually males are the privileged gender and get more attention than the female. Also stroke are less common in females during child bearing time. Age group was found similar in the same study done in Chittagong by Kyastagir et al. regarding risk factors of ischaemic stroke hypertension, diabetes mellitus, cigarette smoking, hyperlipidemia, family history were found among the study population. However, no significant difference was found between two groups regarding the distribution of risk factors. Stroke is a common atherosclerotic vascular disease and above risk factors is common all over the world [22].

\section{Conclusion}

The world health organization defines stroke as "the rapidly developing clinical symptoms and/ or signs of focal (at times global) disturbance of cerebral function, with symptoms lasting more than 24 hours or leading to death with no apparent cause other than that of vascular origin" [24].

Among white population in the USA and Canada, the annual incidence rate is between 1 and 2 per 1000 and the prevalence rate is between 4 and 6 per 1000, but the exact figure depends on the age structure of the population under consideration. Japan has the highest death rate. About $25 \%$ of death of Japanese men and women of all ages are due to CVA, while for the USA and the corresponding figure is around $10 \%$. [25]

In Bangladesh, there is few data on the incidence of and mortally from stroke in the community. In one study in Dhaka medical college hospital, stroke has been shown as the second commonest cause of emergency admission in the medicine wards and constituted about $10-12 \%$ of the total patients in these wards, In another two studies in Chittagong Medical College Hospital and Diabetic Hospital, Dhaka, 2.00 percent and 5.87 percent of the admitted patients, respectively, were suffering from the stroke $[23,26]$.

In patients with acute ischaemic stroke and with motor deficit, the early administration of Fluoxetine with physiotherapy enhanced motor recovery after 3 months.

\section{References}

[1] Frosch MP, Anthony DC, Girolami UD. Central Nervous system. In: Cotran, RS, Kumar V, Fausto N, Nelso F, Robbins SL, Abbas AK(Eds). Robbins and Cotran pathologic basis of disease. $7^{\text {th }}$ ed. 2005 St. Louis, Mo: Elsevier Saunders; 13251419.

[2] Mukherjee D. and Chirag PG. Epidemiology and the Global Burden of Stroke World Neurosurg, 2011, 76, 6S:S85-S90.

[3] Rathor SS, Hinn AR, Cooper LS, Tyroler HA, Rosamond WD. Characterization of incident stroke signs and symptoms: findings from the atherosclerosis risk in communities study. Stroke.2002; 33:2718-2721.

[4] Stewart JC, Cramer SC. Patient-reported measures provide unique insights into motor function after stroke. Stroke. 2013; 44:1111-1116. 
[5] Cools R, Roberts AC, Robbins TW. Serotoninergic regulation of emotional and behavioural control processes. Trends Cogn Sci. $2008 ; 12: 31-40$.

[6] Logue SF, Gould TJ. The neural and genetic basis of executive function: attention, cognitive flexibility, and response inhibition. Pharmacol Biochem Behav. 2011; 123: 45-54.

[7] Cowen P, Sherwood AC. The role of serotonin in cognitive function: evidence from recent studies and implications for understanding depression. J Psycho pharmacol. 2013; 27:575583.

[8] Dam M, Tonin P, De Boni A, Pizzolato G, Casson S, Ermani $\mathrm{M}$, et al. Effects of fluoxetine and maprotiline on functional recovery in poststroke hemiplegic patients undergoing rehabilitation therapy. Stroke. 2006; 27: 1211-1214.

[9] Miyai I, Reding R. Effects of antidepressants on functional recovery following stroke. J Neuro Rehab.2008; 12:5-13.

[10] Pariente J, Loubinoux I,Carel C,Albucher JF,Leger A,Manelfe $\mathrm{C}$,et.al. Fluoxetine modulates motor performance and cerebral activation of patients recovering from stroke. Ann Neurol.2001; 50: 718-729.

[11] Chollet F, Tardy J, Albucher JF, Thalamas C, Berard E, Lamy $\mathrm{C}$, et al. Fluoxetine for motor recovery after acute ischaemic stroke (FLAME): a randomised placebo-controlled trial. Lancet Neurol.2011; 10: 123-130.

[12] Walker FR. A critical review of the mechanism of action for the selective serotonin reuptake inhibitors: do these drugs possess anti-inflammatory properties and how relevant is this in the treatment of depression? Neuropharmacology. 2013; 67: 304-317.

[13] Santarelli L, Saxe M, Gross C, Surget A, Battaglia F, Dulawa $\mathrm{S}$, et. al. Requirement of hippocampal neurogenesis for the behavioral effects of antidepressants. Science. 2003; 301: 805-809. doi: 10.1126/science. 1083328 .

[14] GerdelatMas A, Loubinoux I, Tombari D, Rascol O, Chollet F, Simonetta-Moreau M. Chronic administration of selective serotonin reuptake inhibitor (SSRI) paroxetine modulates human motor cortex excitability in healthy subjects. Neuroimage. 2005; 27: 314-322.

[15] Maya Vetencourt JF, Sale A, Viegi A, Baroncelli L, De
Pasquale R, O'Leary OF, et al. The antidepressant fluoxetine restores plasticity in the adult visual cortex. Science. 2008; 320: 385-388.

[16] Guirado R, Perez-Rando M, Sanchez-Matarredona D,Castrén E, Nacher J. Chronic fluoxetine treatment alters the structure, connectivity and plasticity of cortical interneurons. Int J Neuropsychopharmacol. 2011; 17: 1635-1646.

[17] AclerM, RobolE, FiaschiA, Manganotti P. A double blind placebo RCT to investigate the effects of serotonergic modulation on brain excitability and motor recovery in stroke patients. J.Neurol 2009; 256, 1152-1158.

[18] Michelle AC, Richard F, Josa R, Karem W. Pharmacology, Lippincott's Illustrated Reviews. ${ }^{\text {th }}$ ed.2012; p151-153.

[19] Liepert J. Pharmacotherapy in restorative neurology. Curr Opin Neurol 2008: 183-93.

[20] Lim CM, Kim SW, Park JY, Kim C, Yoon SH, Lee JK. Fluoxetine affords robust neuroprotection in the postischaemic brain via its anti-inflammatory effect. Neurosci Res 2009.

[21] Zittel S, Weiller C, Liepert J: Citalopram improves dexterity in chronic stroke patients. Neurorehabil Neural Repair. 2008, 22: $311-314$.

[22] Muir KW, Buchan A, von Kummer R, Rother J, Baron JC. Imaging of acute stroke. Lancet Neurol 2006; 5: 755-768.

[23] Kayasthagir PK, Hasanuzzaman M, Khondoker MA. 1 Chronic Helicobacter Pylori Infection as A Risk Factor for Acute Ischaemic Cerebral Stroke,Bang jour of Neuro 2010;10:12-22.

[24] Mackay J, Mensah G: The Atlas of Heart Disease and Stroke.Geneva, Switzerland, World Health Organization, 2004.

[25] Feigin VL, Lawes CM, Bennett DA, Anderson CS. Stroke epidemiology: a review of population-based studies of incidence, prevalence, and case-fatality in the late 20th century. Lancet Neurol 2003; 2: 43-53.

[26] Feigin VL, Lawes CM, Bennett DA, Anderson CS. Stroke epidemiology: a review of population-based studies of incidence, prevalence, and case-fatality in the late 20th century. Lancet Neurol 2003; 2: 43-53. 